\title{
Comparison of error-amplification and haptic-guidance training techniques for learning of a timing-based motor task by healthy individuals
}

\author{
Marie-Hélène Milot · Laura Marchal-Crespo • \\ Christopher S. Green · Steven C. Cramer • \\ David J. Reinkensmeyer
}

Received: 12 March 2009/Accepted: 9 September 2009/Published online: 29 September 2009

(c) Springer-Verlag 2009

\begin{abstract}
Performance errors drive motor learning for many tasks. Some researchers have suggested that reducing performance errors with haptic guidance can benefit learning by demonstrating correct movements, while others have suggested that artificially increasing errors will force faster and more complete learning. This study compared the effect of these two techniques-haptic guidance and error amplification-as healthy subjects learned to play a computerized pinball-like game. The game required learning to press a button using wrist movement at the correct time to make a flipper hit a falling ball to a randomly positioned target. Errors were decreased or increased using a robotic device that retarded or accelerated wrist movement, based on sensed movement initiation timing errors. After training with either error amplification or haptic guidance, subjects significantly reduced their timing errors and generalized learning to untrained targets. However, for a subset of more skilled subjects, training with amplified errors produced significantly greater learning than training with the reduced errors associated with haptic guidance, while for a subset of less skilled subjects, training with haptic guidance seemed to benefit learning more. These results suggest that both techniques help enhanced performance of a timing task, but learning is
\end{abstract}

M.-H. Milot $(\bowtie)$ L L. Marchal-Crespo · D. J. Reinkensmeyer Department of Mechanical and Aerospace Engineering, University of California, Irvine, 4200 Engineering Gateway, Irvine, CA 92697, USA

e-mail: mmilot@uci.edu

M.-H. Milot · C. S. Green - S. C. Cramer

Departments of Neurology and Anatomy \& Neurobiology, University of California, Irvine, 843 Health Sciences Road, Hewitt Hall room 1331, Irvine, CA 92697, USA optimized if training subjects with the appropriate technique based on their baseline skill level.

Keywords Motor learning - Timing · Error amplification · Haptic guidance

\section{Introduction}

In order to try to enhance motor learning with robotic devices, two opposite strategies have emerged: haptic guidance and error amplification. The idea behind haptic guidance is to demonstrate correct movement trajectory in order to teach the motor system how to imitate it. It has been hypothesized that haptic guidance provides the motor system with additional proprioceptive and somatosensory cues to help enhance movement planning (Patton and Mussa-Ivaldi 2004). Recent studies have identified specific brain systems that respond to demonstrated movement, such as the mirror neuron system, and play an important role during learning through imitation (for review see Rizzolatti et al. 2009). However, behavioral studies evaluating the impact of haptic guidance during learning of various tasks by healthy or neurologically impaired individuals have yielded inconsistent conclusions. Some studies found that haptic guidance had a positive impact on performance during learning calligraphy (Bluteau et al. 2008), steering a wheel (Marchal-Crespo and Reinkensmeyer 2008b), slalom movements (Wulf and Toole 1999), or learning to move the hand after stroke (Takahashi et al. 2008), whereas other studies have found that haptic guidance did not promote greater learning compared to no assistance (Kahn et al. 2006; O'Malley et al. 2006; Bluteau et al. 2008; Marchal-Crespo and Reinkensmeyer 2008a) or visual demonstration (Feygin et al. 2002; Liu et al. 2006), 
and even hindered learning (Winstein et al. 1994). Furthermore, Marchal-Crespo and Reinkensmeyer (2008a) noted that, during learning of a timing-based task, haptic guidance limited generalization of performance, i.e. that individuals did not generalize the improvement of their timing error to untrained timing actions.

The premise supporting the use of error amplification is related to the fact that many forms of learning are errordriven processes. For example, research on motor adaptation in novel dynamic and kinematic environments suggests that the motor system detects kinematic errors in one trial and proportionally corrects them in the subsequent trial in order to gradually accomplish a skillful performance of the new task (Thoroughman and Shadmehr 2000; Halsband and Lange 2006; Krakauer 2006; Fine and Thoroughman 2007; Franklin et al. 2008). This process has been characterized as the learning of an internal model (Flanagan et al. 1999; Desmurget and Grafton 2000; Seidler et al. 2004; Emken and Reinkensmeyer 2005; Fine and Thoroughman 2007; Tseng et al. 2007; Izawa et al. 2008). By artificially increasing performance error in the course of learning, it has been hypothesized that the motor system could be driven in a way that makes it adapt more completely (Patton et al. 2006b) or quickly (Emken and Reinkensmeyer 2005).

In both healthy and neurologically impaired individuals, studies evaluating the impact of error amplification on performance have found a significant decrease in trajectory error (Patton and Mussa-Ivaldi 2004; Wei and Patton 2004; Wei et al. 2005; Patton et al. 2006a; Grafton et al. 2008; Izawa et al. 2008) as well as a decrease in adaptation time (Emken and Reinkensmeyer 2005; Emken et al. 2007). Improvement in performance following error-amplification training was also found to transfer to other tasks or movement directions (Patton and Mussa-Ivaldi 2004; Wei and Patton 2004; Grafton et al. 2008). However, this has not been shown in post-stroke patients (Patton et al. 2006a). It seems that only one study, by Matsuoka et al. (2007), demonstrated that error amplification provided by visual distortion did not speed up learning during finger pinching in healthy individuals.

Few studies have directly compared the impact of training with haptic guidance or error amplification on learning. Patton et al. (2006b) found that enhancing trajectory errors by the use of force fields induced better learning compared to reducing trajectory errors (haptic guidance) or providing no force field, in individuals with stroke. Using the same paradigm, Cesqui et al. (2008) also suggested that a 2-week training program of errorenhancing trajectory seemed to provide the most benefit to the least impaired individuals, whereas active assistance during target reaching tended to be more helpful for the most impaired individuals. It appears that this result supports the challenge point theory, proposed by Guadagnoli and Lee (2004), which speculated that greater learning is achieved when an optimal challenge is provided to the individuals based on their skill level.

Timing of an action plays a crucial role in the proper accomplishment of many motor skills, such as steering a wheel (Marchal-Crespo and Reinkensmeyer 2008b) or catching or hitting a moving object (Katsumata 2007). Studies found that, with practice, individuals are able to learn to anticipate the correct timing of a task and increase their performance accuracy (Ramnani and Passingham 2001; Feygin et al. 2002; Floyer-Lea and Matthews 2005; Marchal-Crespo and Reinkensmeyer 2008b).

Until now, it seems that no studies have evaluated the relative impact of different forms of robot assistance on learning of this functionally important category of task. Thus, the aim of the present study was to evaluate which training conditions - amplification of error or haptic guidance (reduction of error)—would benefit learning more during a timing-based task. We hypothesized that timing errors drive motor adaptation when learning a timing task, and thus that training with error amplification would provide greater benefit for learning than haptic guidance. We also hypothesized that, as compared to training with haptic guidance, improvement in performance following training with error amplification would generalize to timing actions not part of the training, since subjects would experience a wider range of errors during error-amplification training (Marchal-Crespo and Reinkensmeyer 2008a).

\section{Methodology}

\section{Subjects}

Twenty healthy subjects (12 female; 8 male) between the age of 18-30 years old (mean age $24.0 \pm 2.7$ years) were recruited from the University of California, Irvine (UCI) students and staff. To be included in the study, subjects had to be right handed (Edinburgh handedness questionnaire mean score: $85 \pm 12 \%$ ) and have no active neurological, or orthopedic problem affecting the right upper extremity. Informed consent was obtained from each subject before the evaluation session, and the UC Irvine Institutional Review Board approved the study.

Timing assistive plastic pinball exercise robot (TAPPER)

TAPPER was used in a previous work (Marchal-Crespo and Reinkensmeyer 2008a), but has been modified for the purpose of the current study. TAPPER (see Fig. 1) is a one degree-of-freedom plastic robot actuated by 60 psi air 
Fig. 1 a The computerized pinball game showing the position of the five targets (from left to right: yellow, pink, orange, green, blue), the score sign, the falling ball and the virtual flipper. Note that only 1 target at a time was shown to subjects. b The TAPPER device was actuated by a pneumatic cylinder allowing $5^{\circ}$ of wrist flexion in order for the subjects' fingers to hit the flipperactivating button (Color figure online)
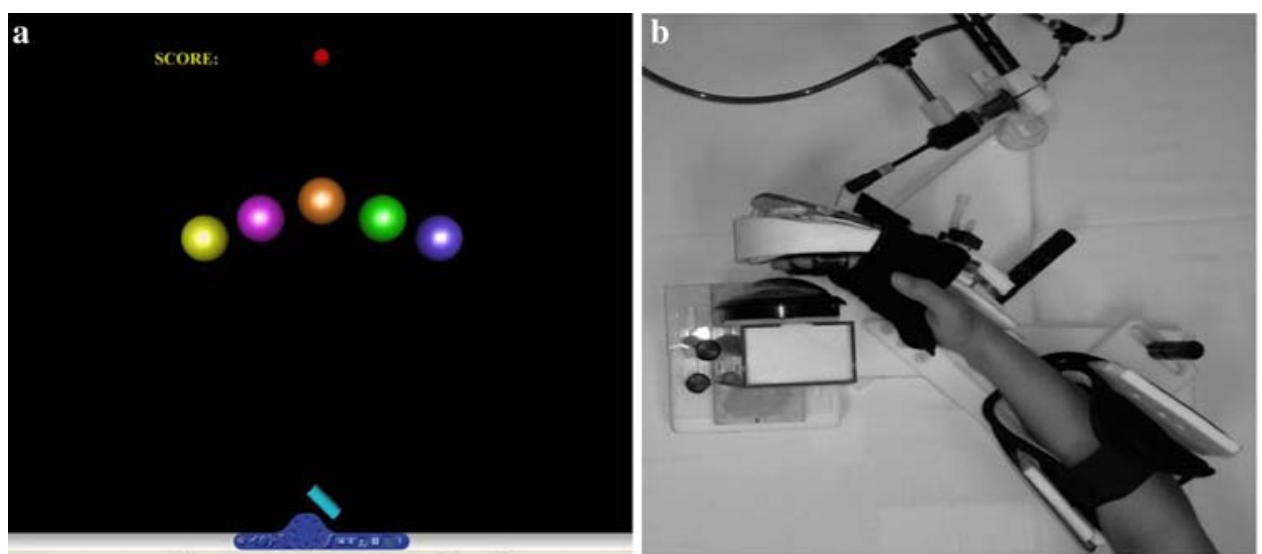

pressure provided by a pressure tank (Chicago Pneumatic; QRS 3.0). TAPPER is composed of a forearm brace mounted on a frame, for positioning of the subject's right forearm during testing, a freely rotating hand brace connected to a pneumatic cylinder, which allows wrist flexion/ extension, and a button that is depressed by the subject's fingers when the hand/robot unit rotates in wrist flexion. A proportional control valve (Festo; MPYE-5-1/8-LF-010-B) and a pressure sensor (SenSym; ASCX100AN) are connected to each of the two chambers of the pneumatic cylinder by plastic tubing. The control valves, the pressure sensors, and the button are connected to an A/D card (Measurement Computing; PCI-DAS1002) and sampled at $1,000 \mathrm{~Hz}$.

For the present experiments, the subject triggered a wrist flexion movement by applying pressure on the locked pneumatic cylinder below a threshold of $\sim 1$ psi. This change of pressure in the pressurized pneumatic cylinder chambers was sensed by the pressure sensors. When the pressure was 1 psi above the actual running maximum value from the pressure reading of the pressurized pneumatic chamber, and following a time delay, the servovalves opened completely in such a way as to drive a rapid wrist flexion. Because the servovalves fully opened on each trial, the speed of movement was kept constant between trials. As the pneumatic cylinder fired, it moved the hand to $5^{\circ}$ of wrist flexion, forcing the subject's fingers to press the button mounted to the TAPPER frame. The signal from the button was then read by the computer and used to actuate the virtual pinball flipper. After a 1-s delay, the valves were then used to return the cylinder and the subject's hand to their original positions.

\section{Pinball simulator}

A pinball simulator was designed using the Real-Time Windows Target and Virtual Reality Toolbox in Simulink (MatWorks ${ }^{\mathrm{TM}}$; Matlab ${ }^{\circledR}$ ) (see Fig. 1), with the distance between the ball start location and the flipper set as $154 \mathrm{~m}$.
The pinball simulator consisted of a ball falling from the top of a computer screen at a constant acceleration of $0.29 \mathrm{~m} / \mathrm{s}^{2}$ towards a flipper positioned at the bottom of the screen. The flipper rotated at a constant radial speed of $30 \mathrm{rad} / \mathrm{s}$. Five color-specific targets were positioned at different locations across the computer screen and presented one at a time at random. The goal of the game was to score points by hitting the selected target. This was done by the subject triggering wrist flexion at the proper timing to make the pneumatic cylinder fire such that the subject's fingers would contact the button. A push on the button would then rotate the flipper seen on the computer screen in order to hit the falling ball towards the target. A hit was considered successful when the ball hit the target at a resolution of $6.9^{\circ}$, which corresponded to a button-press timing accuracy of $4 \mathrm{~ms}$.

\section{Error-amplification and haptic-guidance algorithms}

We wanted to increase or decrease each subject's timing error depending on the training condition. The strategy we used was as follows: to decrease the effect of a timing error (haptic guidance), we delayed the start of robot movement if the subject initiated movement too early, and sped up the start of robot movement if the subject initiated movement too late. To increase the effect of a timing error (error amplification), we sped up the start of robot movement if the subject initiated movement too early, and delayed it in case of a late initiation. We did this in a way that the resulting timing errors were proportional to the original initiation timing errors, with a proportionality constant $k$, which we will call the "error-amplification gain". More specifically, $t=0$ was defined as the time the ball began falling toward the flipper, and $\mathrm{Tb}_{\mathrm{p}}$ defined as the time the subject's fingers pressed the button. Now

$\mathrm{Tb}_{\mathrm{p}}=\mathrm{Ti}_{\mathrm{p}}+D_{\mathrm{c}}+D_{\mathrm{r}}$

where $\mathrm{Ti}_{\mathrm{p}}$ is the time the pressure sensors detect that the subject initiates a wrist movement, and $D_{\mathrm{c}}$ is a programmed 
delay from when the subject initiates movement to when the robot is commanded to move, and $D_{\mathrm{r}}$ is the delay associated with robot movement, from when the robot is commanded to move, to when it actually moves. Note that $D_{\mathrm{r}}$ is a time constant determined by robot properties (25 ms). For a given target, the desired values for the above variables (i.e. those producing a button press that would cause the ball to hit the target perfectly) were defined as $\mathrm{Tb}_{\mathrm{d}}, \mathrm{Ti}_{\mathrm{d}}$ and so

$\mathrm{Tb}_{\mathrm{d}}=\mathrm{Ti}_{\mathrm{d}}+\mathrm{Dc}_{\mathrm{d}}+D_{\mathrm{r}}$

where $T b_{d}$ is the desired time the subject's fingers should press the button to successfully hit the ball to a target, $\mathrm{Ti}_{\mathrm{d}}$ represents the desired time when the subject should initiate a wrist movement and $\mathrm{Dc}_{\mathrm{d}}$ is a constant which we set to be $0.5 \mathrm{~s}$, arbitrarily. Now, the subject's timing error in initiating movement on a trial was defined as $E_{\mathrm{p}}$, so:

$E_{\mathrm{p}}=\mathrm{Ti}_{\mathrm{p}}-\mathrm{Ti}_{\mathrm{d}}$

Substituting Eqs. 1, 2, and 3 to determine the buttonpress timing error $E_{\mathrm{b}}$ yields:

$\mathrm{E}_{\mathrm{b}}=\mathrm{Tb}_{\mathrm{p}}-\mathrm{Tb}_{\mathrm{d}}=\mathrm{E}_{\mathrm{p}}+D_{\mathrm{c}}-\mathrm{Dc}_{\mathrm{d}}$

Now, we wanted the button-press timing error $E_{\mathrm{b}}$ to be proportional to the subject's initiation timing error $E_{\mathrm{p}}$ :

$E_{\mathrm{b}}=k E_{\mathrm{p}}$

where $k$ is the error-amplification gain. Substituting Eq. 4 into Eq. 5 and solving for $D$ c, the programmed delay gives:

$D_{\mathrm{c}}=\mathrm{Dc}_{\mathrm{d}}+E_{\mathrm{p}}(k-1)$

We used Eq. 6 to set the delay between when the subject initiated a movement, and when the robot began to move, in order to proportionally decrease or increase timing error. A $k$ of 1 resulted in no error amplification or haptic guidance; $k>1$ increased timing errors (error amplification); $k<1$ attenuated errors (haptic guidance), and $k=0$ resulted in the subject always hitting the target independent of his timing error (as long as $E_{\mathrm{p}}<\mathrm{Dc}_{\mathrm{d}}$ ). Note that, in the case where a subject initiated a wrist movement very late, with the result that adding the 0.5 -s delay the trial duration was beyond the allocated time frame of $2 \mathrm{~s}$, the cylinder fired without taking into account the 0.5-s delay. This was done to provide consistency of motor and visual input so that the subject would see the flipper rotate on the screen even if he initiated movement way too late.

\section{Adaptive adjustment of game difficulty}

Prior to the introduction of error amplification or haptic guidance, the level of difficulty of the pinball game was adjusted by the nominal error-amplification gain $k$ that each subject experienced during his baseline phase. This was done to ensure a baseline homogeneous game difficulty, to control for the effect of task difficulty on learning. Adjustment was performed based on each subject's skill level as follows:

$$
\begin{aligned}
k(i+1)= & g_{1} \times k(i)-g_{2} \\
& \times\left[w_{1} \times\left(\mathrm{Rs}_{\mathrm{d}}-\mathrm{Rs}_{\mathrm{p}}\right)+w_{2} \times\left|\mathrm{Tb}_{\mathrm{p}}-\mathrm{Tb}_{\mathrm{d}}\right|\right]
\end{aligned}
$$

where $g_{1}$ (1.02) and $g_{2}(0.15)$ represented learning gains, and $w_{1}$ and $w_{2}$ were weighted gains $\left(w_{1}=0.25\right.$ and $\left.w_{2}=0.9\right) . \mathrm{Rs}_{\mathrm{p}}$ and $\mathrm{Rs}_{\mathrm{d}}$ were the subject and desired rate of success $(30 \%)$, respectively, whereas $\mathrm{Tb}_{\mathrm{p}}$ represented the time the subject's fingers actually pressed the button and $\mathrm{Tb}_{\mathrm{d}}$ was the desired time the subject fingers should have pressed the button (from 1.18 to $1.22 \mathrm{~s}$ depending on the target position). Adjustment was made in a preliminary baseline phase (B2 in Fig. 2) in which the subjects played the game for 39 trials. This "game difficulty adjustment phase" started with an error-amplification gain, or $k$ value, of zero, meaning that subjects received full assistance, canceling their timing error, in order for them to

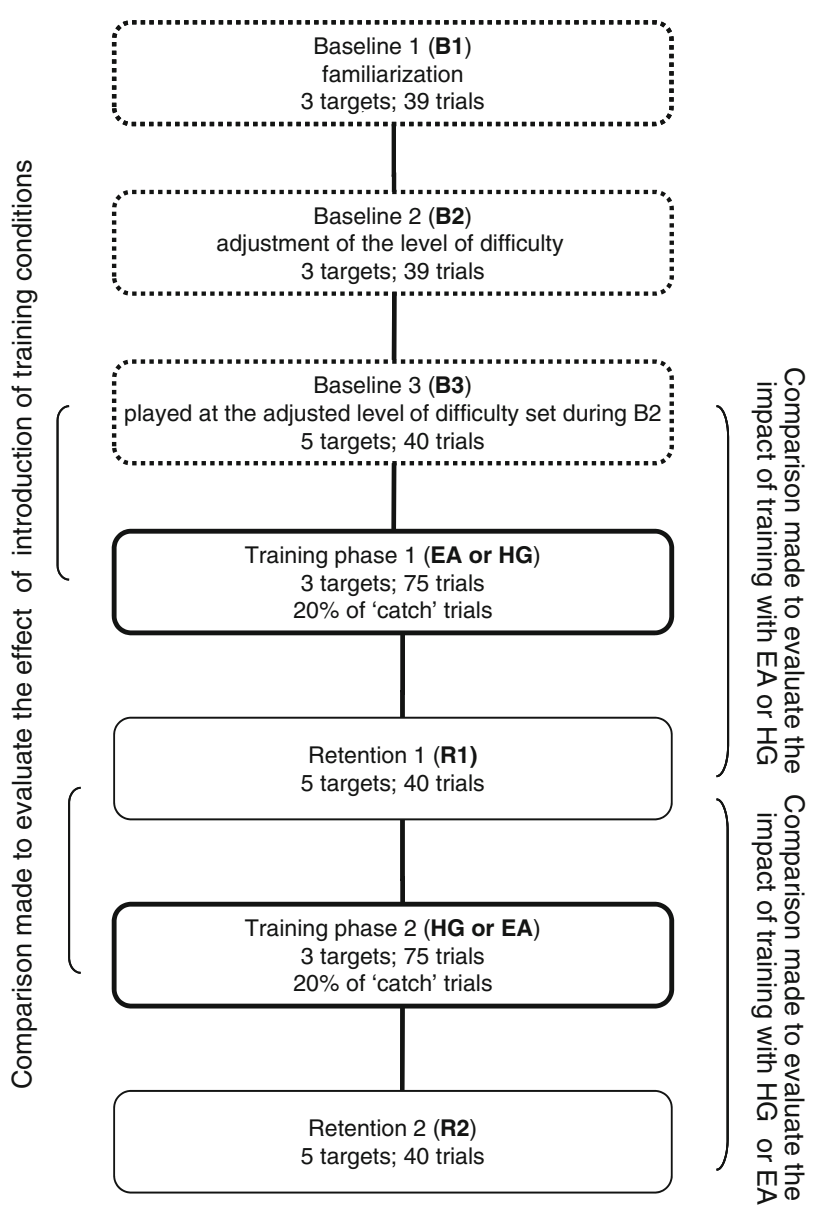

Fig. 2 Study design 
automatically hit the target. The $k$ value was then gradually increased based on the subject's timing error and success rate, until the chosen $30 \%$ rate of success was reached, according to Eq. 7. For subjects for whom the pinball game was challenging, their final $k$ value was thus lower than subjects showing a higher skill level at the game.

The final $k$ value of each subject was increased or decreased by $90 \%$ to provide error amplification or haptic guidance (see Fig. 2), respectively. The $90 \%$ change in the $k$ value was arbitrary, but was chosen to ensure that subjects would experience a difference in error between both training conditions.

Note that game difficulty was adjusted by modifying the $k$ value of each subject instead of modifying the graphics of the game (e.g. size of targets or speed of the ball). Adjusting game difficulty through the game graphics would have translated into changing two variables, game difficulty and graphics, instead of one, game difficulty. Modifying the $k$ value to adjust the game difficulty allowed us to provide a similar game graphic to each subject and to control for the possible confounding effect of varying graphics on learning.

\section{Experimental protocol}

Subjects sat in front of a computer screen. To avoid unwanted finger and hand movements during the game, velcro rings were placed in between the proximal interphalangeal joints and metacarpo-phalangeal joints and at the distal interphalangeal joints while the hand was secured with a strap. TAPPER was secured on the table to allow a $90^{\circ}$ angle at the elbow and the subject's forearm was stabilized with a velcro strap. Care was taken to position the plastic box containing the button used to rotate the flipper in order to ensure that wrist flexion would cause the fingers to touch and activate the button (see Fig. 1).

Subjects were instructed to hit as many targets as possible by moving with the proper timing, taking into account the 0.5-s delay between when they triggered a wrist flexion and when the pneumatic cylinder actually fired. Every time subjects hit a target, a 1-point reward was given and the score was shown at the top of the computer screen along with visual feedback ("Wow! Just on time!"). On missed trials, a feedback message was displayed to inform subjects about their timing error and what action to take on the subsequent trial ("Too early. Hit later!" or "Too late. Hit sooner!"). Out of the five targets, subjects trained with three targets (yellow, orange and blue) shown at random, during error conditions. The two remaining untrained targets (pink and green) were shown only during baseline and retention conditions in order to evaluate generalization. Spatial distribution of targets varied so that subjects would practice different timing of action. A trial lasted $2 \mathrm{~s}$ with an inter-trial pause of two seconds. Position of the next target to be hit was presented to the subjects $1 \mathrm{~s}$ before the beginning of the next trial.

A within-subject cross-over design was used to test the effects of training with error amplification and haptic guidance. The protocol was divided in three parts (see Fig. 2). For the baseline condition, B1 was played using an error-amplification gain ( $k$ value) of 1 , i.e. no error condition was provided. B1 served as familiarization for subjects to become accustomed to the pinball game and the related 0.5-s delay between initiation of a movement and when the robot actually moved the hand towards the button. For B2, the game difficulty adjustment phase, Eq. 7 was used to adjust the level of difficulty of the pinball game based on each subject skill. B3 was played at the adjusted level of difficulty set during B2, and served as the baseline performance assessment for the individualized game difficulty. In between each phase of the baseline conditions, a 1-min pause was provided where subjects could see a performance graph showing timing errors against trials. The total number of trials of the baseline phase was chosen based on a previous study (Marchal-Crespo and Reinkensmeyer 2008a) to ensure that this period would be long enough to allow subjects to reach a plateau in their learning process before being introduced to error amplification or haptic guidance. This was done to evaluate whether introduction to the training conditions would further enhance learning after reaching a steady learning state.

For the training conditions (error amplification and haptic guidance), determination of the starting training condition was randomized and subjects were not aware of which error condition they trained with. Each error condition included $20 \%$ catch trials for which the error condition was unexpectedly removed to ensure that subjects, particularly while training with haptic guidance, would remain alert throughout the entire game, although the same number of catch trials was used for both training conditions for consistency. After the first 40 trials and at the end of the 75 trials, a 1-min pause was provided to the subjects along with their performance graph. The characteristics of the retention phase following training conditions were identical to B3 (see Fig. 2).

\section{Statistical analysis}

For each trial, the timing error was determined by taking the absolute value of the difference between when subjects' fingers contacted the button and the desired time the fingers should have contacted the button. All trials for which the subjects did not apply a sufficient force on the pneumatic cylinder to make it fire or did not trigger a movement with 
their wrist, were recorded and disregarded for data analysis.

Non-normality of the data was observed for the timing error during retention condition following error-amplification training by means of the Kolmogorov-Smirnov test of normality, thus a log transformation was applied. Also, one outlier was removed from the data for comparison of the timing error between B3 and retention conditions.

One-sided paired $t$ tests were used to evaluate: (a) the presence of a learning plateau at the final part of baseline (B3), (b) the effect of introducing error conditions on the subjects timing error, (c) the difference in timing error at the beginning and end of training with each training condition, (d) the difference in timing error between B3 and catch trials during both training conditions, (e) the difference in timing error between B3 and retention conditions for each training condition, and the difference in the change in timing error obtained between both training conditions, for trained and untrained targets, and (f) the formation of an internal model of the delay associated with the triggered movement. Because learning took place during training with error amplification, subjects' performance during the retention phases was compared to the condition they experienced before the training phase. For example, for a subject trained first with error amplification, his performance during R1 was compared to that of B3, whereas comparison of his performance during $\mathrm{R} 2$ was done using R1 as his baseline performance (see Fig. 2). The $P$ value was set at 0.05 . All statistical analyses were performed using $\operatorname{SPSS}^{\circledR}$ software Windows (version 13, Chicago, IL).

\section{Results}

Game difficulty adjustment and reaching of learning plateau

When looking at the last 10 trials of the first phase of the baseline (B1), results showed that subjects reached a mean success rate of $2 \pm 4 \%$. By adjusting game difficulty, a $23 \pm 14 \%$ mean success rate was reached at the beginning (first 10 trials) of the final part of baseline (B3), which remained similar at the end of B3 (last 10 trials) $[t(19)=0.37, P=0.36]$.

Additionally, when looking at the first and last 10 trials of the final baseline phase (B3) in regards to the subjects' timing error, no change was noted meaning that a plateau in the learning process was reached before the introduction of the training conditions $[15 \pm 9$ vs. $18 \pm 15 \mathrm{~ms}$; $t(19)=-1.12, P=0.14]$.
Performance during training with error amplification or haptic guidance

When comparing the timing error during the final baseline phase (B3) to the first 10 trials of the error-amplification (EA) and haptic-guidance (HG) training phases, a significant increase $[14 \pm 9$ vs. $25 \pm 13 \mathrm{~ms} ; t(19)=4.59$, $P=0.001]$ and decrease $[17 \pm 15$ vs. $2 \pm 1 \mathrm{~ms}$; $t(19)=-4.64, P \leq 0.0005]$ in timing error were noted, respectively. Thus, introducing subjects to $\mathrm{EA}$ or $\mathrm{HG}$ conditions produced the desired effect on their performance.

During training with EA, a significant decrease in timing error was noted when comparing the first and last 10 trials of this condition $[25 \pm 13$ vs. $19 \pm 9 \mathrm{~ms} ; t(19)=2.49$, $P=0.01]$, suggesting that subjects learned in the course of error-amplification training. No change in timing error was noted during training with $\mathrm{HG}[2 \pm 1$ vs. $2 \pm 1 \mathrm{~ms}$; $t(19)=-0.48, P=0.32]$. This is not surprising as introduction of the haptic guidance created a floor effect for which subjects' error was already close to zero at the beginning of the error-reduction training, giving small or no room for change (see Fig. 3).

During training with EA, a decrease in subjects' timing error was noted when comparing their B3 performance to their catch trials, i.e. when the training condition was unexpectedly removed, although this decrease did not reach significance $[14 \pm 9$ vs. $12 \pm 6 \mathrm{~ms} ; t(19)=1.79$, $P=0.08$ ]. No change in subjects' timing error between B3 and catch trials was noted during HG $[17 \pm 15$ vs. $15 \pm 12 \mathrm{~ms} ;(19)=1.44, p=0.15]$.

Impact of training with error amplification and haptic guidance on timing error for trained and untrained targets during retention

For the three trained targets, there was a significant decrease in timing error when comparing subjects' baseline performance to that during their retention condition after

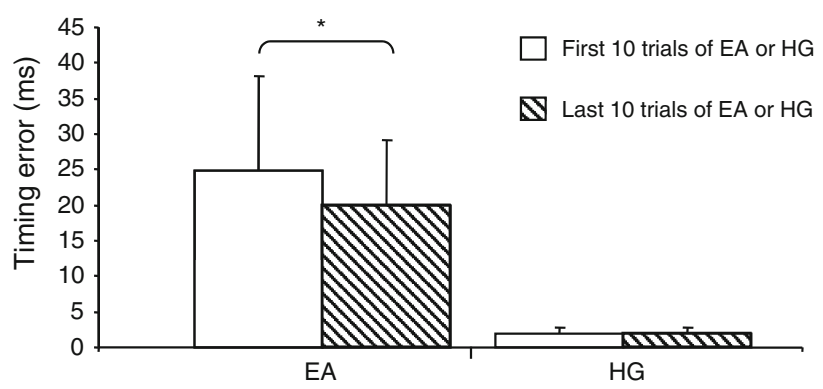

Fig. 3 Comparison of timing error between the first and last 10 trials during training with error amplification $(E A)$ and haptic guidance $(H G)$. Error bars show \pm 1 SD. $* P<0.05$ 
training with EA $[13 \pm 7$ vs. $10 \pm 6 \mathrm{~ms} ; t(18)=3.06$, $P=0.015]$, and HG $[13 \pm 6$ vs. $12 \pm 6 \mathrm{~ms} ; t(18)=1.96$, $P=0.035]$. No significant difference between the change in timing error following EA and HG training was noted $[t(18)=0.81, P=0.47]$. However, by looking at subjects' actual timing error in initiation of movement (see Eq. 6: $E_{\mathrm{p}}$ value), where a negative value was indicative of subjects initiating movement too late and a positive value signifying initiating movement too early, there was significant improvement in subjects' behavior when comparing their baseline performance to that during their retention condition after training with EA $[-42 \pm 53$ vs. $-19 \pm 65 \mathrm{~ms}$; $t(18)=2.01, P=0.017]$. This means that subjects learned to initiate movement earlier in order to be more inclined to push the button on time. No change in subjects' timing error in initiation of movement was noted following training with HG $[-34 \pm 49$ vs. $-34 \pm 58 \mathrm{~ms} ; t(18)=$ $-0.09, P=0.46$ ) [between conditions $t(18)=-2.05$, $P=0.03]$

Significant generalization of performance was noted following training with EA $[13 \pm 8 \mathrm{~ms}$ vs. $11 \pm 6 \mathrm{~ms}$; $t(18)=1.94, P=0.035]$ with generalization approaching significance for $\mathrm{HG}[12 \pm 6 \mathrm{~ms}$ vs. $10 \pm 6 \mathrm{~ms} ; t(18)=$ $1.60, P=0.065]$, but no difference between conditions was noted $[t(18)=0.13, P=0.45]$ (see Fig. 4).
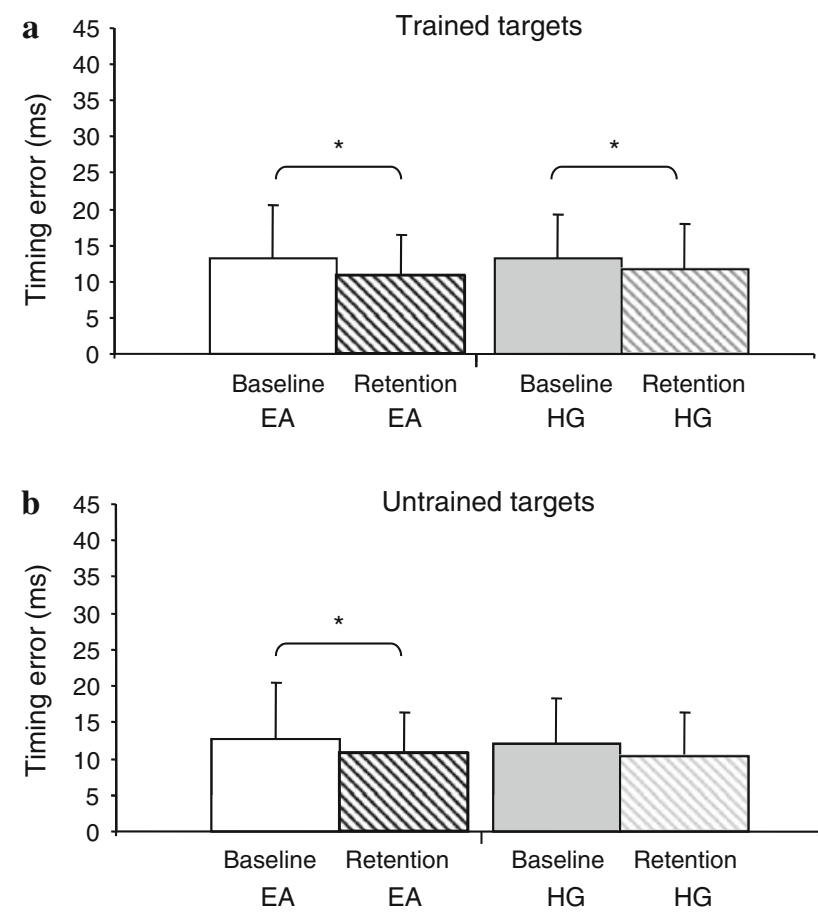

Fig. 4 Comparison of the timing error between baseline and retention conditions following training with error amplification (EA) and haptic guidance $(H G)$ for $\mathbf{a}$ the trained targets, and $\mathbf{b}$ the untrained targets. Baseline condition has been classified based on which training condition each subject experienced first. Error bars show \pm 1 SD. $* P<0.05$
Effect of baseline skill level on relative benefit of error-amplification and haptic-guidance training strategies

A relation between subjects' skill level and related $k$ value was determined by first plotting subjects' timing error and variation of $k$ values during B2, i.e. during the adjustment of game difficulty. The slope of the regression equation of each subject during this phase is indicative of his skill level at the task. In other words, subjects for whom the task was challenging will have a higher slope value (or steeper slope), i.e. greater timing error will be noted across a range of $k$ values, whereas subjects for whom the task was less challenging will show a lower slope value thus less timing error for a range of $k$ values. Next, to determine if the relation between the skill level of subjects and $k$ value could be established, subjects' slope values were plotted against their final $k$ value that was used during B3 and subsequent training conditions (see Fig. 5a). A significant negative relation was noted between these two variables for 16 subjects. In other words, $k$ value could be used as a measure of subjects' skill level at the task where skilled subjects had on average a larger $k$ value at the end of B2 while less skilled subjects had a lower $k$ value.

a
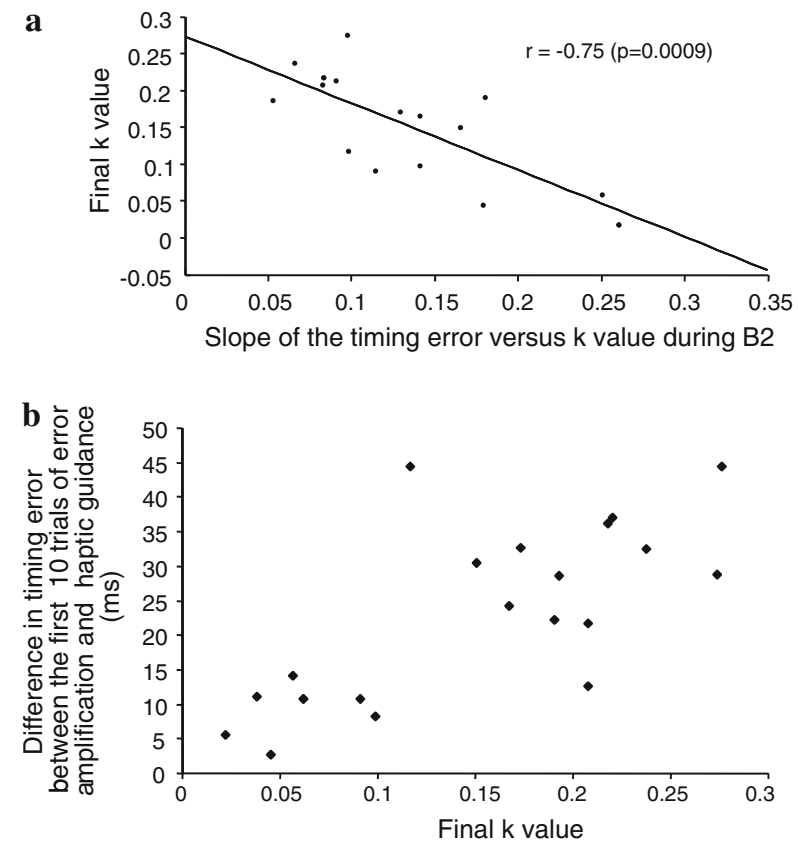

Fig. 5 a Relation between subjects' final $k$ value and value of the slope of the timing error and variation of $k$ value during B2. This slope is a measure of subjects' skill level early in training, with skilled subjects presenting a lower slope value and less skilled subjects presenting a greater slope value. Data are presented for the 16 subjects who showed a significant relation between timing error and variation of $k$ value during B2. b Classification of subjects based on their final $k$ value (skill level). Note that the $y$ axis serves only to better discern the two groups 
Once this relation was determined, a cut off value of subjects' final $k$ value was determined allowing the creation of two distinct skill groups. One group $(n=13)$ had shown a relatively higher skill level during the game difficulty adjustment phase, resulting in a larger final $k$ value $(\geq 0.1)$. Because we adjusted the final $k$ value by $\pm 90 \%$ for error amplification or haptic guidance, and that $k$ was large for this subgroup, the difference in timing error between the two training conditions was large. The other group ( $n=7$ ) had shown a lower skill level during the game difficulty adjustment phase, resulting in a lower final $k$ value $(<0.1)$. Thus, when the $k$ value was changed by the fixed percentages to amplify or decrease errors for this group, there was a smaller resulting difference in timing error between the two training conditions (see Fig. 5b).

Because of the small number of subjects per group, nonparametric analysis, using Wilcoxon signed ranks test, was chosen and performed separately on each of these two groups. For the skilled subjects, training with EA benefited retention more (improvement in timing error of $4 \pm 3 \mathrm{~ms}$; $P=0.002$ ) than training with $\mathrm{HG}$ did (improvement in timing error of $1 \pm 4 \mathrm{~ms} ; P=0.175$ ) (between conditions $P=0.015)$. For this group, generalization of performance was also significant following EA (improvement in timing error of $3 \pm 4 \mathrm{~ms} ; P=0.01$ ), but not following $\mathrm{HG}$ (improvement in timing error of $2 \pm 5 \mathrm{~ms} ; P=0.155$ ), although no significant difference between conditions was observed $(P=0.345)$.

On the other hand, for subjects who had shown a lower skill level in the game difficulty adjustment phase and thus trained with an easier version of the game, training with HG induced better learning (improvement in timing error of $2 \pm 2 \mathrm{~ms}$; $P=0.02$ ) as opposed to training with EA (improvement in timing error of $-0.1 \pm 2 \mathrm{~ms} ; P=0.5$ ), although the difference between conditions only approached significance $(P=0.065)$. No generalization of performance was obtained for that sub-group (improvement following $\mathrm{HG} 1 \pm 3 \mathrm{~ms}$, and following EA $-0.5 \pm 3 \mathrm{~ms} ; P=0.22$ ) (see Fig. 6).

An alternate interpretation of the finding that training technique effectiveness depended on skill level is possible. We subdivided subjects by skill level, as determined by the $k$ value that the adaptive algorithm selected for them in an initial baseline phase (B2). Although it seems clear that the adapted $k$ value is indicative of skill (see Fig. 5a), a confounding consequence of having a small $k$ value was that EA produced a smaller increase in error (see Fig. 5b). This was because we multiplied $k$ by a percentage to amplify error, rather than increasing or decreasing it by a fixed amount. We did this in an attempt to control the relative effects of EA and $\mathrm{HG}$, rather than their absolute effects. However, since subjects with a smaller $k$ value experienced less of an increase in the magnitude of error during EA training, it may be that EA was less effective for these subjects not because they were less skilled, but because EA requires a large increase in error to be effective.

Although we cannot rule out this interpretation with the current data, we think it is unlikely. Subjects with a smaller $k$ value did learn the task, but they learned it when error was reduced (during $\mathrm{HG}$ ). It seems unlikely that they would have an ability to learn the task when errors were reduced, and also when errors were greatly increased (as implied by this alternate interpretation), but not when errors were only moderately amplified (as was the actual case in this study).

By the same line of reasoning, one might argue that $\mathrm{HG}$ was less effective for subjects who trained at a high $k$ value, not because they were more skilled, but because they experienced a relatively large decrease in error when $k$ value was changed. If they had experienced a small error decrease, perhaps they would have learned from HG. This interpretation seems more likely than the alternate interpretation of the ineffectiveness of EA for the less skilled subjects explained above. It may be that decreasing the difficulty of a task slightly opens up learning possibilities which are removed when the task difficulty is decreased too much-when the task becomes too easy. Addressing both of these alternate interpretations will require followup studies, for example, in which subjects are randomized based on skill level into groups that train with different $k$ values (i.e. task difficulties), or in which the size of EA and $\mathrm{HG}$ is controlled in absolute but not relative terms.

Learning a timing-based task involves a gradual construction of an internal model of timing

The first seven subjects were invited back for a follow-up experiment, in which we examined how they learned the timing delay required to successfully play the game. Note that age, handedness and baseline $k$ value at B3 of these subjects did not differ from the other 13 subjects $(P>0.247)$. These seven subjects played the game for a total of 80 trials. The first 40 trials were played as in B1 meaning with $k=1$, and with a nominal delay between movement initiation and robot movement of $\mathrm{Dc}_{\mathrm{d}}=0.5 \mathrm{~s}$. For the last 40 trials, the $k$ value remained the same, but the $\mathrm{Dc}_{\mathrm{d}}$ delay was unexpectedly removed $\left(\mathrm{Dc}_{\mathrm{d}}=0\right)$ meaning that there was no delay between when the subject initiated wrist flexion movement and when the robot moved their hand towards the button. Figure 7 shows the evolution of timing error for these subjects. When comparing the first and last 10 trials of the first 40 trials, results showed that subjects gradually decreased their timing error $[140 \pm 40$ vs. $90 \pm 4 \mathrm{~ms}$; $t(6)=2.32, P=0.03]$, and when the delay was unexpectedly removed, they exhibited a large timing after-effect, as assessed by comparing the last 10 trials of the first condition to the first 10 trials of the second condition [90 \pm 4 vs. 
Fig. 6 Comparison of the difference in timing error (baseline timing error error amplification $(E A)$ and haptic guidance $(H G)$ for the skilled and less skilled subjects. Positive values show improvement in performance while negative values show worsening of performance. Data are presented for the trained and untrained targets. Error bars show \pm 1 SD. $* P<0.05$ retention timing error) between

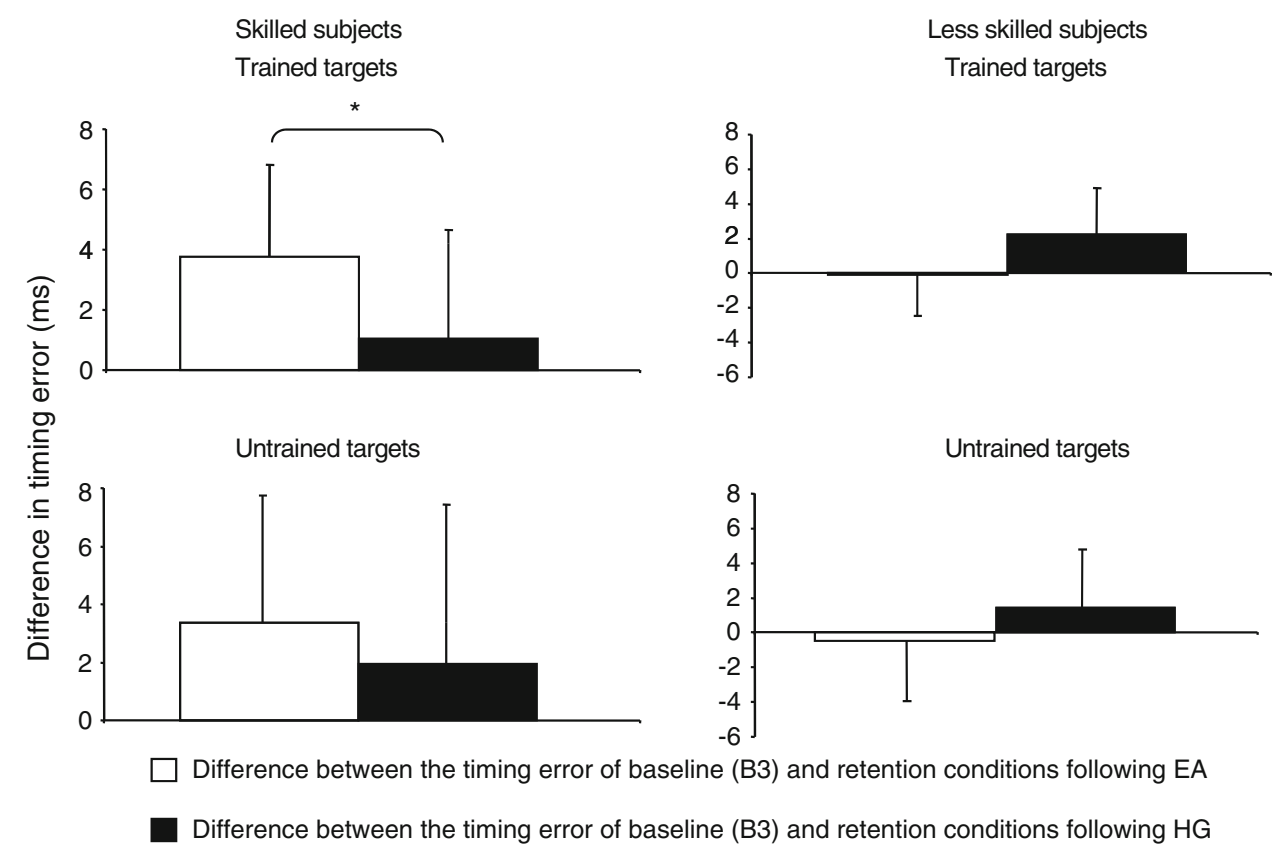

$320 \pm 110 \mathrm{~ms} ; t(6)=-4.47, P=0.002]$. This after-effect took time to wash-out, but at the end of the 80 trials, subjects' timing error returned to a similar value as the one observed for the last 10 trials of the first condition $[90 \pm 4$ vs. $100 \pm 70 \mathrm{~ms} ; t(6)=-0.97, P=0.19]$. These data demonstrate that a key component of learning the pinball-like task was the gradual formation of an internal model of the delay associated with the triggered movement.

\section{Discussion}

This study investigated which training condition-error amplification or haptic guidance-would be more beneficial in learning a timing-based task.
After reaching a steady learning state at the end of the baseline phase, it seemed that demonstrating the correct timing of action or enlarging the timing errors were both relevant strategies that could be used to enhance learning.

This result was not in line with our hypothesis and the results of a previous study by Patton et al. (2006b) which found that error amplification was more beneficial to learning than haptic guidance. Reasons explaining this discrepancy could come from features of the task to be learned and the subjects studied. With respect to the task features, in this study, the subjects had to learn the correct timing of action in order to be successful. Temporal error information had to be integrated by the motor system rapidly since movement at the wrist was short $\left(5^{\circ}\right.$ of wrist
Fig. 7 Evolution of subjects' timing error during learning with a 0.5 -s (condition 1) and 0 -s (condition 2) delay between when subjects triggered a wrist movement and when the robot pneumatic cylinder actually fired

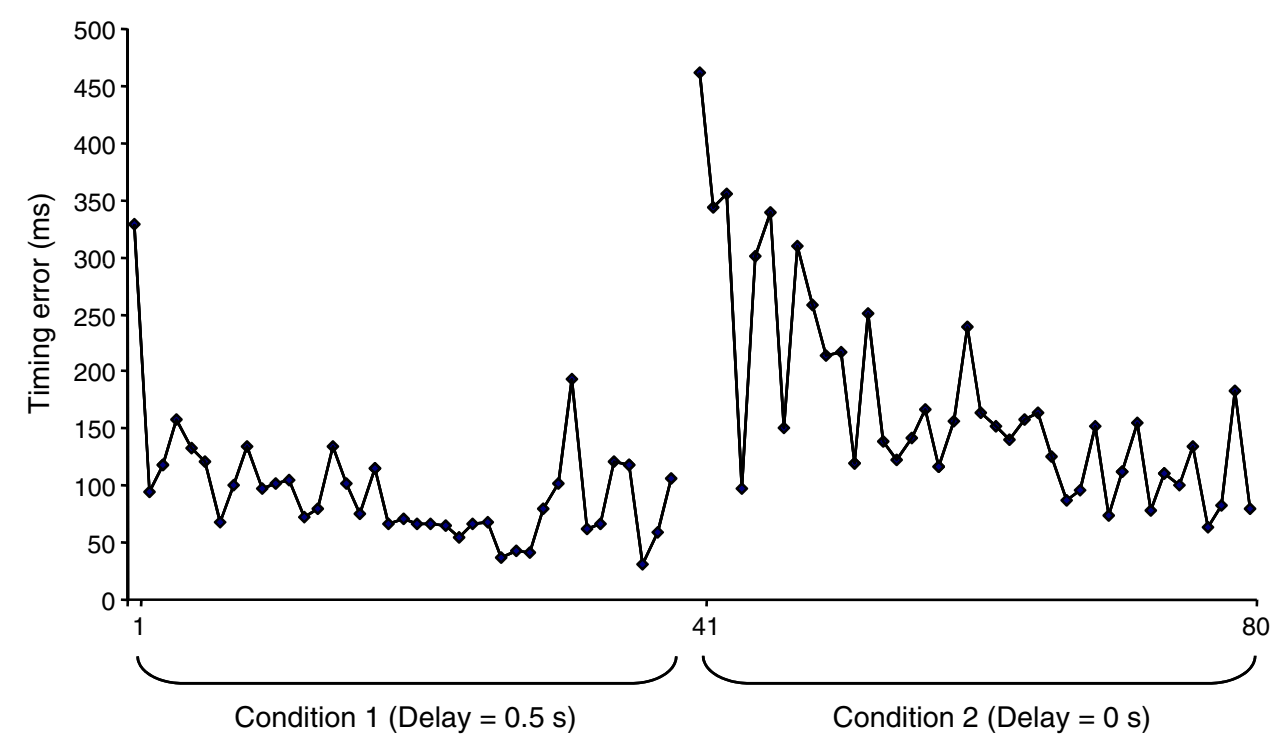

Number of trials 
flexion) as well as the allocated window of timing accuracy $(4 \mathrm{~ms})$. Correction of movement and thus improvement of performance likely occurred primarily by means of feedforward control (Desmurget and Grafton 2000). But in the study of Patton et al. subjects had to adapt to a force field during an error-enhancing trajectory task where integration of motor information, and not timing information, was the central focus. Within this task, these authors only analyzed error in the early movement paths and mainly examined feedforward control mechanism. However, the time subjects took to reach $25 \%$ of the distance to target cannot eliminate the role of feedback control loops during task performance, and learning must have occurred by the combination of feedforward and feedback error controls (Patton et al. 2006b). Additionally, discrepancies between the results of the current study and those of Patton et al. might be akin to distinctive learning mechanisms highlighted for motor and temporal information based on the fact that different brain regions are activated for each respective learning sequence (Sakai et al. 2000, 2002). With respect to subject differences, Patton et al. compared haptic guidance and error-amplification training in individuals with stroke as opposed to healthy individuals as in the current study. Although stroke patients preserve their ability to learn or adapt to a force field (Winstein et al. 1999; Patton et al. 2006b; Takahashi et al. 2008), adaptation can be slower (Dancause et al. 2002) and impairments in the control and execution of the task to learn have been highlighted (Wulf and Toole 1999; Dancause et al. 2002). The impact of these deficits on the learning process remains to be further examined, but the combination of subject and task differences could explain different responses to haptic guidance or error-amplification training.

As subjects pay more attention to a task if it is challenging (Wearden 2004), it was thought that subjects would have been be more engaged in learning during erroramplification training than haptic guidance. On this point, Marchal-Crespo and Reinkensmeyer (2008a) found that, when looking at catch trials during haptic guidance of a timing-based task, subjects' timing error increased, implying that they relied on assistance while learning and were thus possibly less focused on the task. However, the current results do not corroborate these points. Indeed, when comparing subjects' performance during catch trials to that of their baseline (B3), no significant increase in timing error was observed during both training conditions, suggesting that during haptic guidance, subjects did not rely on assistance and seemed to have been as engaged in learning as they were during error-amplification training. Because subjects had to take into consideration a 0.5 -s delay between when they triggered the movement and when the robot actually moved their hand towards the button, and because the performance error was proportional to the subject's timing error, both of which were not the case in the study of Marchal-Crespo and Reinkensmeyer (2008a), the task seems to have been challenging enough to keep subjects' attention throughout the experiment, even during haptic guidance.

Interestingly, while error-amplification training and haptic guidance provided similar gains in motor learning, improvement in performance might arise from different underlying neuroanatomical learning-related circuits. Indeed, the mirror neuron system is suggested to be at the core of action understanding (Binkofski and Buccino 2006) and learning by imitation (Stefan et al. 2005; Buccino et al. 2006; Stefan et al. 2008). Haptic guidance is based on the premise that the appropriate movement or timing of action is taught so that the motor system can imitate or replicate it. Hypothetically, the mirror neuron system might be implicated during such training. Patterns of activity of mirror neurons have been noted principally in pars opercularis of the inferior frontal gyrus (Koski et al. 2002, 2003), but also in premotor cortex (Koski et al. 2002; Kessler et al. 2006), and inferior parietal lobule (Buccino et al. 2004). Activation of these brain areas during training with haptic guidance could enhance the creation of new motor patterns that promote learning (Buccino et al. 2006). On the other hand, in the initial stage of learning a novel task, when unexpected errors are high and task difficulty is increased, activity has been observed in various brain areas involved in error processing (Seidler et al. 2004; Hester et al. 2008). Activity has been noted in areas such as the anterior cingulate cortex (Mars et al. 2005), posterior medial frontal cortex (Hester et al. 2008), and cerebellum (Tseng et al. 2007; Grafton et al. 2008). Hence, by increasing task difficulty, and consequently the occurrence of error, error-amplification training could constantly solicit activity in the error-processing brain areas. That is, introducing larger than normal error in the course of learning could pressure the motor system to continuously update its motor commands and help promote the creation of new ones that benefit learning when the error condition is removed (Patton and Mussa-Ivaldi 2004).

Evaluation of brain regions involved in learning can provide valuable and relevant information on the observed behavioral outcomes (Hester et al. 2008). Until now, it seems that no study has evaluated and compared brain activities following error amplification and haptic guidance trainings and has linked the observed activity to performance of the learned task. The results might have therapeutic implications in terms of tailoring motor training condition to the anatomical location of a focal brain insult such as stroke. A study is in progress to assess this aspect. 
Improvement in performance following erroramplification training and haptic guidance can generalize to untrained targets

Improvement in performance generalized to untrained targets following error-amplification training, with improvement approaching significance for haptic guidance, which corroborate results of previous timing-based (MarchalCrespo and Reinkensmeyer 2008a) and perturbing forcefield studies (Patton and Mussa-Ivaldi 2004; Grafton et al. 2008) in healthy individuals. Based on force-field perturbation studies, it is stated that generalization of performance implies that the motor system has learned an internal model of the perturbing field and generalized it beyond the sets of positions and velocities experienced during the perturbation training to neighboring movements (Patton and MussaIvaldi 2004; Grafton et al. 2008). This might also hold true for timing-based tasks, because our follow-up study showed that the motor system did learn an internal model of the delay associated with the triggered movement, and generalization was noted following error-amplification training. As in Marchal-Crespo and Reinkensmeyer (2008a), generalization of performance to untrained targets was found to be near significant following haptic guidance. MarchalCrespo et al. suggest that the markedly reduced variety of error experiences during haptic guidance could restrict generalization of performance. Expanding upon this, a reduced variety of error experiences could alter the creation of an internal model during training, as it is driven by error signals (Thoroughman and Shadmehr 2000), and consequently limit generalization to untrained targets.

The effectiveness of error-amplification training and haptic guidance depends on starting skill level

It is only after dividing subjects based on their baseline skill level (or $k$ value), that a significant difference in learning was noted between both training conditions. Indeed, it was found that skilled subjects benefited significantly more from error-amplification training than haptic guidance, while haptic guidance helped less skilled subjects relatively more. A similar trend was recently observed in individuals with stroke during the practice of a reaching task using force-field perturbation (Cesqui et al. 2008). The challenge point theory, as described by Guadagnoli and Lee (2004), could help provide an explanation. The authors proposed that greater learning is achieved when an optimal challenge point is reached. One way to achieve this is by providing appropriate task difficulty as the ability of the subject increases. In other words, a task that would be challenging enough for a less skilled subject would be inappropriate for a skilled one. Hence, based on the challenge point theory, learning would be a function of the skill level of subjects and the task difficulty. The current findings can be interpreted in light of this theory. It may be that for the skilled subjects, the increase in their error gain ( $k$ value) during error-amplification training allowed them to reach an optimal challenge point, increasing the potential benefit for learning. As the task difficulty during haptic guidance might not have been challenging enough for these skilled subjects, no learning occurred. On the other hand, for the less skilled subjects, training with error amplification was detrimental to learning due to excess demand level of this condition. Following the Guadagnoli et al. theory, this suggests that there is a limit to interpreting information generated by enhanced errors, which is linked to the individual's information-processing capabilities. Wei et al. (2005), in their study evaluating the impact of visuo-motor distortion on learning, also suggest that great task difficulty creates excessive instability in the adaptation process and no learning occurs, a finding supported by a recent study (Izawa et al. 2008). Although the task in our study differed from that of Wei et al. (2005), it is possible that the increase in the error gain during error-amplification training of the less skilled subjects could have been too large, overwhelming the motor system with a great quantity of new information to process and precluding any improvement in performance. For these subjects, it seems that providing the motor system with haptic guidance, where the motor system could observe the correct timing of movement, might be a better approach.

The fact that both the error-amplification and the hapticguidance trainings seem to favor different subjects, based on their respective baseline level of performance or level of impairments, suggests a strategy for optimizing training. Although more studies are needed to corroborate the present observation, this finding could open the door to the promotion of a more individualized method of teaching motor skills in healthy individuals and those with impairments.

\section{Limitations of the study}

In order for the TAPPER device to provide adequate error amplification or haptic guidance, a constant 0.5-s delay $\left(\mathrm{Dc}_{\mathrm{d}}\right)$ had to be incorporated into the pinball game algorithm, adding a certain amount of difficulty to the learning of the game. Evaluation of subjects' performance without any delay would also be interesting, as greater learning might be expected. In addition, with the algorithm used in this study, true error amplification was not actually provided. True error amplification requires an error gain ( $k$ value) to be greater than 1 , and while the error-amplification paradigm used herein did increase error, it did so with an error gain less than 1 (maximum value of 0.52 ). Still, subjects did experience a significant increase in their 
timing error when introduced to error amplification as compared to their baseline (B3) performance. It could be hypothesized that providing subjects with error gains greater than 1 could have been too demanding and thus detrimental to learning.

\section{Conclusion}

This study evaluated the impact of two different training modes on enhancing learning of a timing-based task: error amplification and haptic guidance. Both training conditions promoted learning. However, when dividing subjects based on their skill level, error-amplification training benefited learning more for the skilled subjects while it seemed that haptic-guidance training was more effective for the less skilled subjects. Additional studies are needed to further validate this observation. Temporal processing during learning is still of great debate. Future studies should explore the brain behavior during learning a timing-based task with error amplification or haptic guidance to gain a better understanding of the related learning processes.

Acknowledgments The project was conducted at the Human Performance Laboratory at the Institute for Clinical and Translational Science University of California, Irvine, with funds provided by the National Center of Research Resources, 5M011 RR-00827-29, US Public Health Service. The project was financed by the National Institute of Health Contract N01-HD-3-3352. Marie-Hélène Milot holds a scholarship from the Canadian Institutes of Health Research and the Fonds de la Recherche en santé du Québec. The authors would like to thank Koyiro Minakata for his technical support.

\section{References}

Binkofski F, Buccino G (2006) The role of ventral premotor cortex in action execution and action understanding. J Physiol Paris 99:396-405

Bluteau J, Coquillart S, Payan Y, Gentaz E (2008) Haptic guidance improves the visuo-manual tracking of trajectories. PLoS ONE 3:e1775

Buccino G, Vogt S, Ritzl A, Fink GR, Zilles K, Freund HJ, Rizzolatti G (2004) Neural circuits underlying imitation learning of hand actions: an event-related fMRI study. Neuron 42:323-334

Buccino G, Solodkin A, Small SL (2006) Functions of the mirror neuron system: implications for neurorehabilitation. Cogn Behav Neurol 19:55-63

Cesqui B, Mazzoleni A, Carrozza M (2008) On the use of divergent force fields in robot-mediated neurorehabilitation. In: IEEE International conference on biomedical robotics and biomechatronics

Dancause N, Ptito A, Levin MF (2002) Error correction strategies for motor behavior after unilateral brain damage: short-term motor learning processes. Neuropsychologia 40:1313-1323

Desmurget M, Grafton S (2000) Forward modeling allows feedback control for fast reaching movements. Trends Cogn Sci 4:423431
Emken JL, Reinkensmeyer DJ (2005) Robot-enhanced motor learning: accelerating internal model formation during locomotion by transient dynamic amplification. IEEE Trans Neural Syst Rehabil Eng 13:33-39

Emken JL, Benitez R, Sideris A, Bobrow JE, Reinkensmeyer DJ (2007) Motor adaptation as a greedy optimization of error and effort. J Neurophysiol 97:3997-4006

Feygin D, Keehner M, Tendick F (2002) Haptic guidance: experimental evaluation of haptic training method for a perceptual motor skill. In: Proceedings of the 10th symposium on haptic interfaces for virtual environment and teleoperator systems

Fine MS, Thoroughman KA (2007) Trial-by-trial transformation of error into sensorimotor adaptation changes with environmental dynamics. J Neurophysiol 98:1392-1404

Flanagan JR, Nakano E, Imamizu H, Osu R, Yoshioka T, Kawato M (1999) Composition and decomposition of internal models in motor learning under altered kinematic and dynamic environments. J Neurosci 19:RC34

Floyer-Lea A, Matthews PM (2005) Distinguishable brain activation networks for short- and long-term motor skill learning. J Neurophysiol 94:512-518

Franklin DW, Burdet E, Tee KP, Osu R, Chew CM, Milner TE, Kawato M (2008) CNS learns stable, accurate, and efficient movements using a simple algorithm. J Neurosci 28:1116511173

Grafton ST, Schmitt P, Van Horn J, Diedrichsen J (2008) Neural substrates of visuomotor learning based on improved feedback control and prediction. Neuroimage 39:1383-1395

Guadagnoli MA, Lee TD (2004) Challenge point: a framework for conceptualizing the effects of various practice conditions in motor learning. J Mot Behav 36:212-224

Halsband U, Lange RK (2006) Motor learning in man: a review of functional and clinical studies. J Physiol Paris 99:414-424

Hester R, Barre N, Murphy K, Silk TJ, Mattingley JB (2008) Human medial frontal cortex activity predicts learning from errors. Cereb Cortex 18:1933-1940

Izawa J, Rane T, Donchin O, Shadmehr R (2008) Motor adaptation as a process of reoptimization. J Neurosci 28:2883-2891

Kahn LE, Zygman ML, Rymer WZ, Reinkensmeyer DJ (2006) Robot-assisted reaching exercise promotes arm movement recovery in chronic hemiparetic stroke: a randomized controlled pilot study. J Neuroeng Rehabil 3:12

Katsumata H (2007) A functional modulation for timing a movement: a coordinative structure in baseball hitting. Hum Mov Sci 26:27-47

Kessler K, Biermann-Ruben K, Jonas M, Siebner HR, Baumer T, Munchau A, Schnitzler A (2006) Investigating the human mirror neuron system by means of cortical synchronization during the imitation of biological movements. Neuroimage 33:227-238

Koski L, Wohlschlager A, Bekkering H, Woods RP, Dubeau MC, Mazziotta JC, Iacoboni M (2002) Modulation of motor and premotor activity during imitation of target-directed actions. Cereb Cortex 12:847-855

Koski L, Iacoboni M, Dubeau MC, Woods RP, Mazziotta JC (2003) Modulation of cortical activity during different imitative behaviors. J Neurophysiol 89:460-471

Krakauer JW (2006) Motor learning: its relevance to stroke recovery and neurorehabilitation. Curr Opin Neurol 19:84-90

Liu J, Cramer SC, Reinkensmeyer DJ (2006) Learning to perform a new movement with robotic assistance: comparison of haptic guidance and visual demonstration. J Neuroeng Rehabil 3:20

Marchal-Crespo L, Reinkensmeyer DJ (2008a) Effect of robotic guidance on motor learning of a timing task. In: IEEE International conference on biomedical robotics and biomechatronics

Marchal-Crespo L, Reinkensmeyer DJ (2008b) Haptic guidance can enhance motor learning of a steering task. J Mot Behav 40:545556 
Mars RB, Coles MG, Grol MJ, Holroyd CB, Nieuwenhuis S, Hulstijn W, Toni I (2005) Neural dynamics of error processing in medial frontal cortex. Neuroimage 28:1007-1013

Matsuoka Y, Brewer BR, Klatzky RL (2007) Using visual feedback distortion to alter coordinated pinching patterns for robotic rehabilitation. J Neuroeng Rehabil 4:17

O’Malley M, Gupta A, Gen M, Li Y (2006) Shared control in haptic systems for performance enhancement and training. J Dyn Syst Meas Control 128:75-85

Patton JL, Mussa-Ivaldi FA (2004) Robot-assisted adaptive training: custom force fields for teaching movement patterns. IEEE Trans Biomed Eng 51:636-646

Patton JL, Kovic M, Mussa-Ivaldi FA (2006a) Custom-designed haptic training for restoring reaching ability to individuals with poststroke hemiparesis. J Rehabil Res Dev 43:643-656

Patton JL, Stoykov ME, Kovic M, Mussa-Ivaldi FA (2006b) Evaluation of robotic training forces that either enhance or reduce error in chronic hemiparetic stroke survivors. Exp Brain Res 168:368-383

Ramnani N, Passingham RE (2001) Changes in the human brain during rhythm learning. J Cogn Neurosci 13:952-966

Rizzolatti G, Fabbri-Destro M, Cattaneo L (2009) Mirror neurons and their clinical relevance. Nat Clin Pract Neurol 5:24-34

Sakai K, Hikosaka O, Takino R, Miyauchi S, Nielsen M, Tamada T (2000) What and when: parallel and convergent processing in motor control. J Neurosci 20:2691-2700

Sakai K, Ramnani N, Passingham RE (2002) Learning of sequences of finger movements and timing: frontal lobe and action-oriented representation. J Neurophysiol 88:2035-2046

Seidler RD, Noll DC, Thiers G (2004) Feedforward and feedback processes in motor control. Neuroimage 22:1775-1783

Stefan K, Cohen LG, Duque J, Mazzocchio R, Celnik P, Sawaki L, Ungerleider L, Classen J (2005) Formation of a motor memory by action observation. J Neurosci 25:9339-9346
Stefan K, Classen J, Celnik P, Cohen LG (2008) Concurrent action observation modulates practice-induced motor memory formation. Eur J Neurosci 27:730-738

Takahashi CD, Der-Yeghiaian L, Le V, Motiwala RR, Cramer SC (2008) Robot-based hand motor therapy after stroke. Brain 131:425-437

Thoroughman KA, Shadmehr R (2000) Learning of action through adaptive combination of motor primitives. Nature 407:742-747

Tseng YW, Diedrichsen J, Krakauer JW, Shadmehr R, Bastian AJ (2007) Sensory prediction errors drive cerebellum-dependent adaptation of reaching. J Neurophysiol 98:54-62

Wearden JH (2004) Decision processes in models of timing. Acta Neurobiol Exp (Wars) 64:303-317

Wei Y, Patton JL (2004) Force field training to facilitate learning visual distortions: a sensory crossover experiment. In: Proceedings of the 12th International symposium on haptic interfaces for virtual environment and teleoperator systems

Wei Y, Bajaj P, Scheidt R, Patton JL (2005) Visual error augmentation for enhancing motor learning and rehabilitative relearning. In: Proceedings of the IEEE 9th International conference on rehabilitation robotics

Winstein CJ, Pohl PS, Lewthwaite R (1994) Effects of physical guidance and knowledge of results on motor learning: support for the guidance hypothesis. Res Q Exerc Sport 65:316-323

Winstein CJ, Merians AS, Sullivan KJ (1999) Motor learning after unilateral brain damage. Neuropsychologia 37:975-987

Wulf G, Toole T (1999) Physical assistance devices in complex motor skill learning: benefits of a self-controlled practice schedule. Res Q Exerc Sport 70:265-272 